Arq. Bras. Med. Vet. Zootec., v.70, n.3, p.965-974, 2018

\title{
Composição de ácidos graxos do queijo e leite de vacas alimentadas com casca de banana
}

[Fatty acid composition of cheese and milk of cows fed with banana peel]

\author{
M.T.P. Melo ${ }^{1}$, V.R. Rocha Júnior ${ }^{1 *}$, P.R.S. Pimentel ${ }^{1}$, L.A. Caldeira ${ }^{1}$, J.R.M. Ruas ${ }^{1}$, J.M.A. Chamone ${ }^{1}$, \\ F. Vieira e Silva ${ }^{1}$, D.P.D. Lanna ${ }^{2}$, C. Soares ${ }^{1}$ \\ ${ }^{1}$ Universidade Estadual de Montes Claros - Janaúba, MG \\ ${ }^{2}$ Universidade Estadual de São Paulo - Piracicaba, SP
}

\begin{abstract}
RESUMO
Objetivou-se avaliar os níveis de casca de banana seca ao sol na dieta de vacas F1 Holandês x Zebu sobre o perfil de ácidos graxos do leite e do queijo minas frescal. Os tratamentos foram constituídos de 0,15 , 30,45 e $60 \%$ de substituição da silagem de sorgo pela casca de banana. O delineamento experimental foi em dois quadrados latinos 5 x 5 simultâneos. As amostras de leite e queijo foram analisadas quanto ao perfil de ácidos graxos por cromatografia gasosa. Observou-se efeito quadrático para o somatório de ácidos graxos poli-insaturados do leite, com valor máximo no nível de $23,54 \%$ de substituição. Houve efeito linear decrescente para os ácidos graxos C15:0 iso e C16:0 iso. Os ácidos graxos heneicosanoico, linoleico, linoleico conjugado e araquidônico apresentaram efeito quadrático. Não houve efeito das dietas sobre os ácidos graxos no queijo. A substituição de até $60 \%$ da silagem de sorgo por casca de banana na dieta de vacas em lactação pode ser alternativa para produção e processamento do leite, quando se considera a melhora no valor nutricional da fração lipídica do leite e o aumento dos teores de ácido linoleico conjugado.
\end{abstract}

Palavras-chave: ácido linoleico conjugado, qualidade do leite, subprodutos, vacas F1 Holandês x Zebu

\begin{abstract}
This study aimed to evaluate the inclusion levels of dry banana peel in the sun in the diet of F1 Holstein $x$ Zebu cows on the profile of fatty acids of milk and Minas fresh cheese. The diets were composed of 0, 15, 30, 45, and $60 \%$ replacement of sorghum silage by banana peel. The experimental design was Latin squares, simultaneous, $5 \times$ 5. Samples of milk and cheese were analyzed for fatty acid composition by gas chromatography. Quadratic effect for the sum of milk polyunsaturated fatty acids, with the maximum level of 23,54\% replacement. There was a decreasing linear effect for C15:0 iso fatty acids and C16: 0 iso. The heneicosanoic fatty acids, linoleic, conjugated linoleic and arachidonic presented quadratic effect. There was no effect of diets on fatty acids in cheese. Replacement of up to $60 \%$ of the sorghum silage of a banana peel in the diet of cows can be an alternative to milk production and processing when considering the improvement in the nutritional value of the milk lipid fraction and increase of the levels of conjugated linoleic acid.
\end{abstract}

Keywords: conjugated linoleic acid, milk quality, by-products, F1 Holstein x Zebu cows

\section{INTRODUÇÃO}

A modificação do perfil lipídico da gordura do leite, por meio de manipulação da dieta de vacas, é praticada há bastante tempo com o intuito de aumentar o equilíbrio entre os teores ácidos

Recebido em 28 de setembro de 2016

Aceito em 4 de agosto de 2017

* Autor para correspondência (corresponding author)

E-mail: vicente.rocha@unimontes.br graxos saturados e insaturados, tendo em vista os efeitos benéficos dessa alteração na dieta sobre a saúde em humanos (Siri-Tarino et al., 2010; Santos et al., 2013; Lanier e Corl, 2015). Um ácido graxo que tem sido frequentemente abordado em pesquisas que visam a seu aumento na gordura do leite é o ácido linoleico conjugado 
(CLA), especialmente seu isômero no leite, o ácido rumênico (C18:2 cis-9, trans-11) e seu precursor para síntese endógena na glândula mamária, que é o ácido vaccênico (C18:1 trans11) (García et al., 2010; Mourthé et al., 2015). Dentre os possíveis efeitos benéficos deste ácido graxo à saúde humana, destaca-se sua atuação como imunomodulador, na diminuição da gordura corporal e como anticarcinogênico (Dilzer e Park, 2012; Lucatto et al., 2014).

A casca de banana apresenta teor de gordura que varia de 2 a 10,9\% de extrato etéreo (Mahopatra et al., 2010), com mais de 40\% dos ácidos graxos totais sendo representados pelos ácidos graxos poli-insaturados, linoleico e $\alpha$-linolênico, que têm efeito benéfico sobre os lipídeos sanguíneos (Emaga et al., 2007). A introdução desses ácidos graxos poli-insaturados na dieta pode ainda favorecer a bio-hidrogenação ruminal e, consequentemente, a produção dos intermediários desse processo no rúmen, como o CLA (Menezes et al., 2010).

De acordo com Nudda et al. (2014), o processamento do leite não causa uma mudança substancial no perfil de ácidos graxos e, portanto, as concentrações de ácidos graxos na gordura de produtos do leite são essencialmente dependentes do perfil de ácidos graxos do leite antes do seu processamento.

Portanto, o aproveitamento da casca de banana na alimentação de vacas em lactação apresenta potencial para alterar o perfil de ácidos graxos do leite e do queijo, o que pode os tornar alimentos mais nutritivos e benéficos à saúde humana, além de ser uma alternativa importante ao descarte desse subproduto no meio ambiente. Objetivouse, por meio deste trabalho, avaliar os níveis da casca de banana seca ao sol em substituição à silagem de sorgo na dieta de vacas F1 Holandês $\mathrm{x}$ Zebu sobre o perfil de ácidos graxos do leite e do queijo minas frescal.

\section{MATERIAL E MÉTODOS}

O experimento foi conduzido na Fazenda Experimental da Universidade Estadual de Montes Claros - Unimontes, localizada no município de Janaúba, no norte de Minas Gerais. $\mathrm{O}$ projeto de pesquisa foi aprovado pela Comissão de Ética em Experimentação e BemEstar Animal da Unimontes, sob o parecer
081/2014. Foram utilizadas 10 vacas F1 Holandês x Zebu, com $70 \pm 11$ dias de lactação ao início do experimento. $\mathrm{O}$ delineamento experimental foi em dois quadrados latinos $5 \mathrm{X}$ 5, simultâneos, compostos, cada um de cinco animais, cinco tratamentos e cinco períodos experimentais. Foram utilizadas cinco dietas, sendo: silagem de sorgo sem a inclusão da casca de banana (controle); inclusão de 15, 30, 45 e $60 \%$ da casca de banana em substituição à silagem de sorgo. A substituição da silagem de sorgo pela casca de banana foi feita com base na matéria seca. A relação volumoso:concentrado das dietas foi de 70:30. O experimento teve duração de 80 dias, divididos em cinco períodos de 16 dias, sendo os primeiros 12 dias para adaptação dos animais às dietas e os quatro últimos dias para coleta de dados e amostras. As dietas foram formuladas para serem isoproteicas, para vacas com média de $500 \mathrm{~kg}$ de peso corporal e produção média de $15 \mathrm{~kg}$ de leite corrigido para $3,5 \%$ de gordura $\mathrm{dia}^{-1}$, e foram fornecidas para as vacas duas vezes ao dia, às sete horas e às $14 \mathrm{~h}$, em sistema de dieta completa.

As cascas de banana eram oriundas de frutas maduras do cultivar Prata-Anã e apresentavam teor médio de matéria seca de $10,32 \%$ e $6,25 \%$ de extrato etéreo. As cascas foram previamente desidratas por exposição ao sol por $12 \pm 3$ dias. Após o processo de desidratação, foram trituradas em picadeira estacionária, a fim de se obterem partículas de 3 a 4 centímetros, e armazenadas em sacos de náilon em galpão coberto.

Os alimentos ofertados eram pesados em balança digital e o fornecimento foi ajustado de forma que as sobras representassem $5 \%$ da quantidade de matéria seca fornecida. A proporção dos ingredientes utilizados nas dietas e a composição química delas encontram-se na Tab. 1. As vacas foram mantidas em baias individuais $e$ ordenhadas com ordenhadeira mecânica duas vezes ao dia, às oito horas e às $15 \mathrm{~h}$. Utilizou-se a presença do bezerro para estimular a decida do leite, e, após a ordenha, estes permaneceram com as mães para mamada do leite residual por, aproximadamente, 30 minutos. Amostras de leite de cada animal foram coletadas duas vezes ao dia, nos últimos quatro dias de cada período, sendo feito um pool das amostras do leite da ordenha da manhã e da tarde, proporcionalmente à quantidade produzida de manhã e à tarde. Com 
o leite produzido no último dia de cada período experimental, o queijo minas frescal foi fabricado no Laboratório de Tecnologia de Produtos de Origem Animal da Unimontes Campus Janaúba, de acordo com a técnica recomendada por Furtado e Lourenço Neto (1994). Nas amostras de leite e queijo minas frescal, foram determinados os teores de gordura pelo método de Gerber.

Amostras de leite, queijo e da casca de banana seca ao sol foram encaminhadas ao Laboratório de Nutrição e Crescimento Animal da ESALQ/Piracicaba para determinação do perfil de ácidos graxos. As amostras transmetiladas foram analisadas em cromatógrafo a gás, modelo Focus CG-Finnigan, com detector de ionização de chama, coluna capilar CP-Sil 88 (Varian), com $100 \mu \mathrm{m}$ de comprimento por $0,25 \mu \mathrm{m}$ de diâmetro interno e $0,20 \mu \mathrm{m}$ de espessura do filme. Os ácidos graxos foram identificados por comparação dos tempos de retenção dos ésteres metílicos das amostras com padrões de ácidos graxos de manteiga. Os ácidos graxos foram quantificados por normalização das áreas dos ésteres metílicos. Os resultados dos ácidos graxos foram expressos em $\mathrm{mg} / \mathrm{g}$ de gordura.

A qualidade nutricional da fração lipídica do leite e do queijo foi avaliada pelos dados de composição em ácidos graxos, empregando-se os seguintes cálculos: índice de aterogenecidade $(\mathrm{IA})=$ aterogenecidade $(\mathrm{IA})=\{(\mathrm{C} 12: 0+(4 \times$ C14:0) $+\mathrm{C} 16: 0)\} /($ Lácidos graxos monoinsaturados $+\Sigma \omega 6+\Sigma \omega 3)$ e índice de trombogenicidade $(\mathrm{IT})=(\mathrm{C} 14: 0+\mathrm{C} 16: 0+$ $\mathrm{C} 18: 0) /\{(0,5 \times \quad \Sigma$ Ácidos graxos monoinsaturados $)+(0,5 \times \Sigma \omega 6+(3 \times \Sigma \omega 3)+$ $(\Sigma \omega 3 / \Sigma \omega 6)\}$, segundo Ulbricth e Southage (1991); razão entre ácidos graxos hipocolesterolêmicos e hipercolesterolêmicos $(\mathrm{HH})=$ (monoinsaturado + poliinsaturado $) /(\mathrm{C} 14: 0+\mathrm{C} 16: 0)$ e ácidos graxos desejáveis $(\mathrm{AGD})=$ (insaturados $+\mathrm{C} 18: 0$ ), segundo Costa et al. (2008); razão entre ácidos graxos poli-insaturados e ácidos graxos saturados e razão entre $\omega 6$ e $\omega 3$ (Costa et al., 2008).

Tabela 1. Proporção dos ingredientes das dietas experimentais (\%) e composição química das dietas, na base da matéria seca

\begin{tabular}{cccccc}
\hline \multirow{2}{*}{ Ingredientes } & \multicolumn{5}{c}{ Níveis de substituição da casca de banana (\% MS) } \\
\cline { 2 - 6 } & 0 & 15 & 30 & 45 & 60 \\
\hline Silagem de sorgo & 70 & 59,5 & 49 & 38,5 & 28 \\
Casca de banana & 0 & 10,5 & 21 & 31,5 & 42 \\
Farelo de soja & 17,31 & 17,29 & 17,27 & 17,24 & 17,22 \\
Milho moído & 11,73 & 11,75 & 11,77 & 11,8 & 11,82 \\
Suplemento mineral ${ }^{1}$ & 0,96 & 0,96 & 0,96 & 0,96 & 0,96 \\
\hline \multicolumn{5}{c}{ Composição química } \\
\hline Matéria seca & 50,55 & 56,26 & 61,97 & 67,68 & 73,39 \\
Matéria mineral & 6,64 & 7,33 & 8,02 & 8,71 & 9,4 \\
Proteína bruta & 13,65 & 13,89 & 14,12 & 14,35 & 14,58 \\
Extrato etéreo & 2,83 & 3,28 & 3,74 & 4,19 & 4,64 \\
${ }^{2}$ CNF & 20,70 & 22,16 & 23,61 & 25,07 & 26,52 \\
${ }^{3}$ FDNcp & 56,38 & 53,55 & 50,72 & 47,90 & 45,07 \\
Lignina & 7,51 & 7,59 & 7,66 & 7,74 & 7,82 \\
\hline
\end{tabular}

${ }^{1}$ Níveis de garantia por $\mathrm{kg}$ de produto: cálcio (128g mín)(157g máx), fósforo (100g mín), sódio (120g mín), magnésio $(15 \mathrm{~g})$, enxofre $(33 \mathrm{~g})$, cobalto $(135 \mathrm{mg})$, cobre $(2160 \mathrm{mg})$, ferro $(938 \mathrm{mg})$, iodo $(160 \mathrm{mg})$, manganês $(1800 \mathrm{mg})$, selênio $(34 \mathrm{mg})$, zinco $(5760 \mathrm{mg})$, flúor $(1000 \mathrm{mg}) ;{ }^{2} \mathrm{CNF}=$ carboidratos não fibrosos; ${ }^{3} \mathrm{FDNcP}=$ fibra em detergente neutro corrigida para cinza e proteína.

Os dados obtidos foram submetidos à análise de variância, utilizando-se o programa Sisvar e, quando significativas, as médias dos tratamentos foram comparadas pelo teste de Dunnet e submetidas à análise de regressão, tendo-se considerado $\alpha=0,05$.

\section{RESULTADOS E DISCUSSÃO}

Conforme Tab. 2, não houve alteração nos teores de gordura do leite com a substituição da silagem de sorgo pela casca de banana seca ao sol, apresentando média geral de 4,76\%. O nível de produção das vacas, sua genética e relação 
volumoso:concentrado da dieta $(70: 30)$ podem ajudar a explicar o alto teor de gordura do leite. Segundo Reis et al. (2012), a produção relaciona-se ao efeito de diluição, já a genética de vacas mestiças de raças leiteiras influencia na produção de leite com maior teor de gordura. Não houve efeito das dietas sobre o somatório dos ácidos graxos saturados (AGS) e monoinsaturados (AGMI) do leite (Tab. 2). Os ácidos graxos mais representativos na classe dos AGS foram o palmítico (C16:0) e o mirístico (C14:0). Esses ácidos graxos, juntamente com o láurico $(\mathrm{C} 12: 0)$, têm sido associados a doenças coronarianas, como a arteriosclerose, pois contribuem para aumentar a concentração das lipoproteínas de baixa densidade (Santos et al., 2013). Por outro lado, em estudo em que foram analisados os efeitos de ácidos graxos dietéticos sobre a prevenção secundária de doenças cardíacas coronarianas, não houve evidência na redução do risco dessas doenças com a substituição de ácidos graxos saturados por insaturados (Schwingshackl e Hoffmann, 2014).

Houve efeito linear decrescente para os ácidos graxos C15:0 iso e C16:0 iso (Tab. 2). Resultado semelhante foi obtido por Ribeiro et al. (2014), para o somatório de ácidos graxos de cadeia ímpar e ramificada no leite de vacas recebendo dietas com níveis crescentes de óleo de girassol. Mourthé et al. (2015), quando trabalharam com vacas Holandês x Gir em pastagem de capimmarandu, também observaram redução linear desses ácidos graxos com a inclusão de grão de soja na dieta. Esses ácidos graxos são oriundos, principalmente, dos micro-organismos ruminais que os sintetizam após mudanças na biossíntese dos lipídeos da dieta. Nesse sentido, esses ácidos graxos podem ser indicativos de mudanças na população da microbiota ruminal (Vlaeminck et al., 2006). Ainda, segundo estes autores, bactérias celulolíticas contêm maiores quantidades de ácidos graxos iso, enquanto as amilolíticas contêm em sua membrana maiores teores de ácidos graxos anteiso e de cadeia linear ímpar. Os menores teores de FDN observados para as dietas com maiores níveis de inclusão de casca de banana (Tab. 1) contribuem para reforçar essa hipótese.
Houve efeito quadrático para o ácido graxo C21:0. Boa parte dos ácidos graxos saturados de cadeia longa é proveniente da dieta, os quais são transportados como triglicerídeos por meio de lipoproteínas de muito baixa densidade (VLDL) e absorvidos pelo epitélio do tecido mamário, sendo essa ação dependente da lipase lipoproteica presente nesse tecido (Lanier e Corl, 2015). Essa hipótese é corroborada pela proporção razoável de ácidos graxos saturados de cadeia longa $(14,72 \%)$ presentes na casca de banana. Para os demais ácidos graxos saturados, não houve diferença entre as dietas estudadas. Entre os ácidos graxos monoinsaturados (AGMI), aquele que predominou na gordura do leite foi o ácido oleico (C18:1 c9), representando $64,61 \%$ do total de AGMI do leite. Efeitos anticolesterolêmicos são atribuídos ao ácido oleico, o que o torna importante sob a ótica nutricional do leite (Lanier e Corl, 2015). O ácido vaccênico não foi influenciado pelas dietas. Esse ácido possui uma importância sob o ponto de vista nutricional do leite, pois constitui o principal precursor do CLA cis-9, trans-11 no tecido mamário de ruminantes. Ademais, há indicações de que o próprio ácido vaccênico tem efeito protetor contra arteriosclerose (Bassett et al., 2010).

Houve efeito quadrático para o somatório de ácidos graxos poli-insaturados (Tab. 2). O ponto de máxima concentração desses ácidos graxos no leite foi obtido com o nível de $23,54 \%$ de casca de banana, não tendo sido observada diferença entre os tratamentos e a testemunha $(0 \%$ de casca). Lanier e Corl (2015) citam que os ácidos graxos poli-insaturados presentes na gordura do leite são derivados dos ácidos graxos do plasma sanguíneo, os quais têm origem em ácidos graxos livres da mobilização de gordura corporal e dos ácidos graxos de origem dietética transportados como triglicerídeos pelas lipoproteínas de muito baixa densidade. De acordo com Chilliard et al. (2007), como os AGPI não são sintetizados pelos tecidos dos ruminantes, sua concentração no leite é determinada pela quantidade desses ácidos que chegam ao duodeno. 
Tabela 2. Teor de gordura (\%) e perfil de ácidos graxos da gordura do leite ( $\mathrm{mg} /$ grama de gordura) de vacas F1 Holandês x Zebu alimentadas com ou sem inclusão de casca de banana, com respectivas equações de regressão (ER), erro-padrão da média (EPM) e valor de $\mathrm{P}(\operatorname{Pr}>\mathrm{fc})$

\begin{tabular}{|c|c|c|c|c|c|c|c|c|}
\hline \multirow{2}{*}{ Componentes } & \multicolumn{5}{|c|}{ Níveis de casca de banana } & \multirow[b]{2}{*}{ ER } & \multirow[b]{2}{*}{ EPM } & \multirow[b]{2}{*}{$\mathrm{Pr}>\mathrm{Fc}$} \\
\hline & 0 & 15 & 30 & 45 & 60 & & & \\
\hline Gordura & 4,73 & 4,87 & 4,64 & 4,61 & 4,96 & $\hat{\mathrm{Y}}=4,76$ & 0,067 & 0,6695 \\
\hline$\sum$ AGS1 & 75,4985 & 75,2149 & 77,2018 & 76,5650 & 75,1947 & $\hat{\mathrm{Y}}=75,93$ & 0,4037 & 0,7070 \\
\hline $\mathrm{C} 4: 0$ & 2,9204 & 2,8467 & 3,0226 & 3,1295 & 2,9977 & $\hat{\mathrm{Y}}=2,98$ & 0,0478 & 0,1822 \\
\hline C6:0 & 2,2435 & 2,1668 & 2,3996 & 2,4857 & 2,3361 & $\hat{\mathrm{Y}}=2,32$ & 0,0562 & 0.1158 \\
\hline C8:0 & 1,3415 & 1,3369 & 1,5767 & 1,5205 & 1,4790 & $\hat{Y}=1,45$ & 0,0482 & 0,6201 \\
\hline $\mathrm{C} 10: 0$ & 3,4041 & 3,4220 & 3,3465 & 3,1445 & 4,1030 & $\hat{Y}=3,48$ & 0,1624 & 0,1834 \\
\hline $\mathrm{C} 11: 0$ & 0,1102 & 0,1131 & 0,1630 & 0,1740 & 0,1485 & $\hat{Y}=0,14$ & 0,0129 & 0,2364 \\
\hline $\mathrm{C} 12: 0$ & 3,7958 & 3,7644 & 3,7415 & 3,8028 & 3,3831 & $\hat{Y}=3,69$ & 0,0794 & 0,9442 \\
\hline $\mathrm{C} 13: 0$ iso & 0,0367 & 0,0314 & 0,0371 & 0,0337 & 0,0347 & $\hat{Y}=0,03$ & 0,0010 & 0,7316 \\
\hline $\mathrm{C} 13: 0$ anteiso & 0,1423 & 0,1211 & 0,1452 & 0,1425 & 0,1522 & $\hat{Y}=0,14$ & 0,0052 & 0,1437 \\
\hline C13:0 & 0,2312 & 0,2126 & 0,2594 & 0,2672 & 0,2709 & $\hat{Y}=0,24$ & 0,0113 & 0,3680 \\
\hline $\mathrm{C} 14: 0$ iso & 0,1374 & 0,1164 & 0,1273 & 0,1221 & 0,1163 & $\hat{Y}=0,12$ & 0,0039 & 0,6917 \\
\hline C14:0 & 11,4941 & 13,3206 & 10,8905 & 10,4365 & 11,7732 & $\hat{Y}=11,58$ & 0,4928 & 0,3001 \\
\hline $\mathrm{C} 15: 0$ iso & 0,3641 & 0,3373 & 0,3141 & 0,3082 & $0,2801^{*}$ & 1 & 0,0142 & 0,0489 \\
\hline C15:0 anteiso & 0,4594 & 0,3987 & 0,4318 & 0,4198 & 0,3962 & $\hat{\mathrm{Y}}=0,42$ & 0,0116 & 0,8158 \\
\hline C15:0 & 1,1083 & 0,9538 & 1,1195 & 1,1305 & 1,0506 & $\hat{Y}=1,08$ & 0,0327 & 0,2969 \\
\hline $\mathrm{C} 16: 0$ iso & 0,2053 & 0,1937 & 0,1753 & $0,1378 *$ & $0,1334 *$ & 2 & 0,0145 & 0,0221 \\
\hline C16:0 & 38,3174 & 36,8191 & 40,3289 & 40,1586 & 36,9690 & $\hat{\mathrm{Y}}=38,52$ & 0,7516 & 0,0707 \\
\hline $\mathrm{C} 17: 0$ iso & 0,2710 & 0,2936 & 0,2554 & 0,2363 & 0,2385 & $\hat{Y}=0,26$ & 0,0107 & 0,0633 \\
\hline C17:0 & 0,6543 & 0,6470 & 0,5341 & 0,6403 & 0,5834 & $\hat{Y}=0,61$ & 0,0231 & 0,6194 \\
\hline C18:0 & 8,0120 & 7,9321 & 8,0633 & 7,9939 & 8,5036 & $\hat{Y}=8,10$ & 0,1028 & 0,8614 \\
\hline C20:0 & 2,9204 & 2,8467 & 3,0226 & 3,1295 & 2,9977 & $\hat{Y}=2,98$ & 0,0021 & 0,7238 \\
\hline $\mathrm{C} 21: 0$ & 2,2435 & 2,1668 & 2,3996 & 2,4857 & 2,3361 & 3 & 0,0016 & 0,0027 \\
\hline C22:0 & 1,3415 & 1,3369 & 1,5767 & 1,5205 & 1,4790 & $\hat{Y}=1,45$ & 0,0022 & 0,3561 \\
\hline C23:0 & 3,4041 & 3,4220 & 3,3465 & 3,1445 & 4,1030 & $\hat{Y}=3,48$ & 0,0005 & 0,9668 \\
\hline $\mathrm{C} 24: 0$ & 0,1102 & 0,1131 & 0,1630 & 0,1740 & 0,1485 & $\hat{Y}=0,14$ & 0,0012 & 0,9568 \\
\hline$\Sigma$ AGMI2 & 21,8819 & 22,4825 & 20,5094 & 20,9937 & 22,0160 & $\hat{Y}=21,58$ & 0,3596 & 0,7520 \\
\hline $\mathrm{C} 10: 1$ & 0,3658 & 0,3790 & 0,4600 & 0,4568 & 0,3658 & $\hat{\mathrm{Y}}=0,40$ & 0,0052 & 0,9720 \\
\hline C12:1 & 0,0964 & 0,0884 & 0,1100 & 0,1248 & 0,0978 & $\hat{\mathrm{Y}}=0,10$ & 0,0026 & 0,1534 \\
\hline C14:1c9 & 1,2816 & 1,3280 & 1,5446 & 1,5500 & 1,1946 & $\hat{Y}=1,38$ & 0,0251 & 0,5379 \\
\hline C16:1c9 & 2,0644 & 1,9146 & 2,2708 & 2,2632 & 1,8440 & $\hat{Y}=2,07$ & 0,0271 & 0,8258 \\
\hline C17:1 & 0,2162 & 0,1472 & 0,1748 & 0,1698 & 0,1612 & $\hat{Y}=0,17$ & 0,0071 & 0,2893 \\
\hline C18:1 c9 & 14,9196 & 11,7878 & 13,4570 & 13,3620 & 12,1588 & $\hat{\mathrm{Y}}=13,13$ & 0,3180 & 0,1538 \\
\hline C18:1 trans & 1,2354 & 1,0648 & 1,1278 & 1,0404 & 1,0028 & $\hat{Y}=1,09$ & 0,0347 & 0,7401 \\
\hline C18:1 c11 & 0,5998 & 0,4972 & 0,5196 & 0,4516 & 0,5388 & $\hat{\mathrm{Y}}=0,52$ & 0,0492 & 0,4947 \\
\hline C18:1 c12 & 0,3292 & 0,2676 & 0,2862 & 0,2486 & 0,2816 & $\hat{\mathrm{Y}}=0,28$ & 0,0246 & 0,3969 \\
\hline C18:1 c13 & 0,1824 & 0,1512 & 0,1602 & 0,1222 & 0,1236 & $\hat{\mathrm{Y}}=0,15$ & 0,0167 & 0,2377 \\
\hline C18:1 t16 & 0,1130 & 0,0998 & 0,1010 & 0,1074 & 0,0800 & $\hat{\mathrm{Y}}=0,10$ & 0,0056 & 0,7912 \\
\hline C18:1 c15 & 0,0342 & 0,0280 & 0,0324 & 0,0326 & 0,0236 & $\hat{Y}=0,03$ & 0,0051 & 0,3973 \\
\hline C20:1 & 0,0066 & 0,0074 & 0,0104 & 0,0082 & 0,0086 & $\hat{\mathrm{Y}}=0,008$ & 0,0067 & 0,6684 \\
\hline $\mathrm{C} 22: \ln 9$ & 0,0050 & 0,0074 & 0,0066 & 0,0058 & 0,0088 & $\hat{\mathrm{Y}}=0,006$ & 0,0038 & 0,3384 \\
\hline$\Sigma$ AGPI3 & 2,0375 & 1,8106 & 1,8291 & 2,0169 & 2,2844 & 4 & 0,0859 & 0,0133 \\
\hline $\mathrm{C} 18: 2 \mathrm{c} 9 \mathrm{c} 12$ & 1,2146 & 1,1740 & 1,3446 & 1,4122 & 1,3210 & 5 & 0,0735 & 0,0251 \\
\hline C18:3 n6 & 0,0038 & 0,0074 & 0,0100 & 0,0104 & 0,0114 & $\hat{\mathrm{Y}}=0,008$ & 0,0005 & 0,3372 \\
\hline C18:3 n3 & 0,2610 & 0,2234 & 0,2866 & 0,2740 & 0,2656 & $\hat{\mathrm{Y}}=0,26$ & 0,0052 & 0,2351 \\
\hline C18:2 c9t11 & 0,3028 & 0,3284 & $0,3374 *$ & 0,3160 & 0,2586 & 6 & 0,0191 & 0,0447 \\
\hline $\mathrm{C} 20: 2$ & 0,0044 & 0,0066 & 0,0012 & 0,0034 & 0,0052 & $\hat{\mathrm{Y}}=0,004$ & 0,0005 & 0,9315 \\
\hline C20:3 n6 & 0,0104 & 0,0120 & 0,0206 & 0,0140 & 0,0156 & $\hat{\mathrm{Y}}=0,01$ & 0,0016 & 0,5646 \\
\hline C20:4 n6 & 0,0826 & 0,0682 & 0,1050 & 0,0878 & 0,0882 & 7 & 0,0033 & 0,0252 \\
\hline $\mathrm{C} 22: 2$ & 0,0012 & 0,0026 & 0,0012 & 0,0006 & 0,0006 & $\hat{\mathrm{Y}}=0,001$ & 0,0002 & 0,6418 \\
\hline $\mathrm{C} 20: 5 \mathrm{n} 3$ & 0,0232 & 0,0192 & 0,0222 & 0,0158 & 0,0146 & $\hat{\mathrm{Y}}=0,019$ & 0,0017 & 0,2284 \\
\hline $\mathrm{C} 22: 5$ & 0,0466 & 0,0390 & 0,0526 & 0,0484 & 0,0510 & $\hat{\mathrm{Y}}=0,047$ & 0,0013 & 0,6856 \\
\hline $\mathrm{C} 22: 6 \mathrm{n} 3$ & 0,0178 & 0,0114 & 0,0004 & 0,0032 & 0,0042 & $\hat{\mathrm{Y}}=0,007$ & 0,0033 & 0,9120 \\
\hline
\end{tabular}

$1 \hat{\mathrm{Y}}=0,356760-0,001238 \mathrm{x}(\mathrm{R} 2=0,9744) ; 2 \hat{\mathrm{Y}}=0,209-0,0013 \mathrm{x}(\mathrm{R} 2=0,9477) ; 3 \hat{\mathrm{Y}}=0,014877+0,000025 \mathrm{x}-0.000003 \times 2$ $(\mathrm{R} 2=0,8053) ; 4 \hat{\mathrm{Y}}=2,018377-0,017232 \mathrm{x}+0.000366 \times 2(\mathrm{R} 2=0,9820) ; 5 \hat{\mathrm{Y}}=1,201489-0,010010 \mathrm{x}+0.000255 \times 2(\mathrm{R} 2=$ $0,9922) ; 6 \hat{\mathrm{Y}}=0.337234-0,006059 \mathrm{x}+0.000085 \mathrm{x} 2(\mathrm{R} 2=0,6729) ; 7 \hat{\mathrm{Y}}=0,068260+0,000635 \mathrm{x}(\mathrm{R} 2=0,7897) ; *$ diferem da testemunha pelo teste Dunnett; 1. ácidos graxos saturados; 2. ácidos graxos monoinsaturados; 3. ácidos graxos poliinsaturados. 
Houve feito quadrático para o ácido linoleico (C18:2 cis9cis12) no leite. Esse ácido graxo foi predominante entre os ácidos graxos poliinsaturados, e o ponto máximo de sua concentração no leite foi com o nível de 19,63\% de substituição de silagem de sorgo por casca de banana. Este ácido graxo, além de apresentar efeitos de prevenção a doenças cardivasculares, é um dos precursores do CLA no leite (Lucatto et al., 2014). O ácido linoleico conjugado CLA (C18:2 cis9trans11) encontrado no leite teve efeito quadrático pela inclusão de casca de banana na dieta, tendo alcançado valor máximo na substituição de $35,64 \%$ da silagem de sorgo por casca de banana. A dieta com $30 \%$ de casca de banana apresentou diferença significativa para este ácido graxo no leite quando comparado à dieta testemunha. Segundo Lanier e Corl (2015), o CLA no leite tem origem tanto da biohidrogenação parcial dos ácidos graxos no rúmen quanto da atividade da enzima $\Delta^{9}$ desaturase na glândula mamária sobre o ácido vaccênico (C18:1trans11). Considerando que não houve efeito das dietas sobre os teores de ácidos vaccênicos no leite, presume-se que a maior parte do CLA C18:2cis9trans11 seja advinda da incompleta bio-hidrogenação ocorrida no rúmen, sobre a qual os taninos presentes na casca de banana podem ter influenciado (Toral et al., 2011; Lahlou et al., 2014). Tal pressuposição é corroborada pelo aumento nos teores de ácido linoleico (C18:2cis9) no leite com a inclusão de casca de banana nas dietas. Emaga et al. (2007) relataram a presença de taninos nas cascas de banana. Estudos apontam sobre a contribuição favorável dos taninos na alimentação de ruminantes com relação aos efeitos na biohidrogenação de AGPI pelas bactérias ruminais (Toral et al., 2011). Benchaar et al. (2008) detectaram mudanças na composição da gordura do leite quando suplementaram a dieta de vacas com $6,7 \mathrm{~g} . \mathrm{Kg}^{-1}$ com extrato de tanino condensado de quebracho.

O teor de ácido araquidônico (C20:4n6) no leite apresentou efeito linear crescente em função do nível de casca de banana na dieta das vacas. O ácido araquidônico é precursor de eicosanoides produzidos na célula, os quais podem atenuar respostas pró-inflamatórias e pró-agregatórias, contribuindo para diminuição dos riscos de arteriosclerose (Ferrucci et al., 2006). Não foi observada a influência nos somatórios dos AGS, AGMI, AGPI $(\mathrm{P}>0,05)$ do queijo pelas dietas estudadas (Tab. 3). Os teores de ácido $\gamma$ linolênico (C18:3n6) encontrados nos queijos produzidos com leite de vacas que receberam dietas contendo $60 \%$ de casca de banana foram maiores em comparação à dieta sem casca. $\mathrm{O}$ ácido $\gamma$-linolênico (C18:3n6) é considerado não essencial, pois é sintetizado a partir do ácido linoleico, por meio da enzima $\Delta 6$-desaturase, e desempenha papel na manutenção da estrutura e da função da membrana celular, na regulação da síntese e no transporte de colesterol e como precursor de eicosanoides, incluindo prostaglandinas e leucotrienos que atuem na redução de processos inflamatórios (Oliveira e Nunes-Pinheiro, 2013).

Os ácidos mirístico (C14:0) e palmítico (C16:0) foram os mais presentes nos queijos entre os demais ácidos graxos saturados. É importante destacar a presença do ácido esteárico (C18:0), que é o principal produto da bio-hidrogenação completa dos ácidos graxos poli-insaturados dentro do rúmen e sempre está presente em quantidades consideráveis no leite e em seus derivados (Chilliard et al., 2007). O ácido oleico foi predominante entre os AGMI, representando $64,4 \%$ do total de ácidos graxos monoinsaturados.

De acordo com Nudda et al. (2014), o processamento do leite não causa mudança significativa no perfil de ácidos graxos e, portanto, as concentrações de ácidos graxos na gordura de produtos do leite são essencialmente dependentes do perfil de ácidos graxos do leite antes do seu processamento. Apesar de não terem sido verificados os mesmos efeitos das dietas das vacas nos ácidos graxos do leite e do queijo, observa-se uma semelhança entre os valores médios de ácidos graxos do leite antes e após seu processamento.

Não houve efeito das dietas sobre os índices de aterogenicidade, trombogenecidade, relação de ácidos graxos hipo e hipercolesterolêmicos e ácidos graxos desejáveis do leite, bem como sobre os índices nutricionais da gordura do queijo (Tab. 4). 
Tabela 3. Teor de gordura (\% do extrato seco total) e perfil de ácidos graxos da gordura do queijo minas frescal (mg / grama de gordura) fabricado com leite de vacas F1 Holandês x Zebu alimentadas com ou sem inclusão de casca de banana, com respectivas equações de regressão (er), erro-padrão da média (EPM) e valor de $\mathrm{P}(\mathrm{Pr}>\mathrm{fc})$

\begin{tabular}{|c|c|c|c|c|c|c|c|c|}
\hline \multirow[t]{2}{*}{ Componentes } & \multicolumn{6}{|c|}{ Níveis de casca de banana } & \multirow[b]{2}{*}{ EPM } & \multirow[b]{2}{*}{$\operatorname{Pr}>\mathrm{Fc}$} \\
\hline & 0 & 15 & 30 & 45 & 60 & $\overline{E R}$ & & \\
\hline Gordura & 35,20 & 27,86 & 36,16 & 34,54 & 31,39 & $\hat{\mathrm{Y}}=33,03$ & 1,520 & 0.7200 \\
\hline$\sum \mathrm{AGS} 1$ & 74,73 & 78,90 & 75,92 & 76,37 & 78,61 & $\hat{\mathrm{Y}}=76,91$ & 0,8020 & 0,6775 \\
\hline $\mathrm{C} 4: 0$ & 2,7698 & 2,9490 & 2,7430 & 2,7210 & 2,9934 & $\hat{\mathrm{Y}}=2,83$ & 0,0565 & 0,5006 \\
\hline C6:0 & 2,1682 & 2,4182 & 2,1934 & 2,2064 & 2,6186 & $\hat{Y}=2,32$ & 0,0868 & 0,3985 \\
\hline C8:0 & 1,3342 & 1,2746 & 1,4924 & 1,4970 & 1,3522 & $\hat{\mathrm{Y}}=1,39$ & 0,0446 & 0,2207 \\
\hline $\mathrm{C} 10: 0$ & 3,3046 & 2,9802 & 3,6856 & 3,7084 & 3,2860 & $\hat{Y}=3,39$ & 0,1369 & 0,2056 \\
\hline $\mathrm{C} 11: 0$ & 0,0856 & 0,0802 & 0,1038 & 0,1114 & 0,0998 & $\hat{\mathrm{Y}}=0,09$ & 0,0058 & 0,4568 \\
\hline $\mathrm{C} 12: 0$ & 4,1034 & 3,7010 & 4,5868 & 4,6444 & 4,1122 & $\hat{Y}=4,22$ & 0,1745 & 0,1993 \\
\hline $\mathrm{C} 13: 0$ iso & 0,0518 & 0,0474 & 0,0494 & 0,0554 & 0,0478 & $\hat{\mathrm{Y}}=0,05$ & 0,0015 & 0,4669 \\
\hline $\mathrm{C} 13: 0$ anteiso & 0,1514 & 0,1572 & 0,1694 & 0,1868 & 0,1566 & $\hat{\mathrm{Y}}=0,16$ & 0,0064 & 0,5139 \\
\hline $\mathrm{C} 13: 0$ & 0,1538 & 0,1508 & 0,1802 & 0,1818 & 0,1580 & $\hat{\mathrm{Y}}=0,16$ & 0,0067 & 0,6289 \\
\hline $\mathrm{C} 14: 0$ iso & 0,1364 & 0,1250 & 0,1386 & 0,1384 & 0,1048 & $\hat{\mathrm{Y}}=0,12$ & 0,0065 & 0,0669 \\
\hline $\mathrm{C} 14: 0$ & 11,8938 & 11,7082 & 13,0572 & 13,1430 & 11,4098 & $\hat{Y}=12,24$ & 0,3588 & 0,1392 \\
\hline $\mathrm{C} 15: 0$ iso & 0,3678 & 0,3582 & 0,3734 & 0,3472 & 0,2848 & $\hat{\mathrm{Y}}=0,34$ & 0,0160 & 0,2014 \\
\hline C15:0 anteiso & 0,4478 & 0,4392 & 0,4754 & 0,4542 & 0,3700 & $\hat{\mathrm{Y}}=0,43$ & 0,0179 & 0,2672 \\
\hline $\mathrm{C} 15: 0$ & 1,1868 & 1,2082 & 1,2796 & 1,2760 & 1,1626 & $\hat{\mathrm{Y}}=1,22$ & 0,0237 & 0,7628 \\
\hline $\mathrm{C} 16: 0$ iso & 0,2126 & 0,1810 & 0,2056 & 0,2010 & 0,1674 & $\hat{\mathrm{Y}}=0,19$ & 0,0084 & 0,3474 \\
\hline $\mathrm{C} 16: 0$ & 37,4306 & 41,8248 & 37,3352 & 37,8298 & 41,0414 & $\hat{\mathrm{Y}}=39,09$ & 0,9672 & 0,5119 \\
\hline $\mathrm{C} 17: 0$ iso & 0,3828 & 0,3384 & 0,3388 & 0,3318 & 0,3352 & $\hat{\mathrm{Y}}=0,34$ & 0,0094 & 0,9971 \\
\hline $\mathrm{C} 17: 0$ & 0,4492 & 0,4094 & 0,4624 & 0,3692 & 0,3106 & $\hat{\mathrm{Y}}=0,40$ & 0,0277 & 0,6742 \\
\hline C18:0 & 7,8576 & 8,2294 & 6,8008 & 6,6994 & 8,2960 & $\hat{\mathrm{Y}}=7,57$ & 0,3460 & 0,1678 \\
\hline C20:0 & 0,1040 & 0,1238 & 0,0988 & 0,0970 & 0,1122 & $\hat{\mathrm{Y}}=0,10$ & 0,0049 & 0,1233 \\
\hline $\mathrm{C} 21: 0$ & 0,0056 & 0,0148 & 0,0040 & 0,0102 & 0,0094 & $\hat{Y}=0,008$ & 0,0019 & 0,1481 \\
\hline $\mathrm{C} 22: 0$ & 0,0708 & 0,0972 & 0,0896 & 0,0922 & 0,1012 & $\hat{\mathrm{Y}}=0,09$ & 0,0052 & 0,8499 \\
\hline C23:0 & 0,0254 & 0,0360 & 0,0218 & 0,0306 & 0,0336 & $\hat{\mathrm{Y}}=0,02$ & 0,0026 & 0,3323 \\
\hline C24:0 & 0,0430 & 0,0536 & 0,0438 & 0,0422 & 0,0524 & $\hat{\mathrm{Y}}=0,04$ & 0,0025 & 0,5509 \\
\hline$\sum$ AGMI2 & 22,4242 & 18,5732 & 21,1646 & 20,7730 & 18,6542 & $\hat{Y}=20,31$ & 0,7474 & 0,6646 \\
\hline$\overline{\mathrm{C}} 10: 1$ & 0,3658 & 0,3790 & 0,4600 & 0,4568 & 0,3658 & $\hat{\mathrm{Y}}=0,40$ & 0,0217 & 0,1936 \\
\hline $\mathrm{C} 12: 1$ & 0,0964 & 0,0884 & 0,1100 & 0,1248 & 0,0978 & $\hat{Y}=0,10$ & 0,0064 & 0,1837 \\
\hline C14:1c9 & 1,2816 & 1,3280 & 1,5446 & 1,5500 & 1,1946 & $\hat{\mathrm{Y}}=1,37$ & 0,0717 & 0,2930 \\
\hline C16:1c9 & 2,0644 & 1,9146 & 2,2708 & 2,2632 & 1,8440 & $\hat{Y}=2,07$ & 0,0874 & 0,3932 \\
\hline C17:1 & 0,2162 & 0,1472 & 0,1748 & 0,1698 & 0,1612 & $\hat{\mathrm{Y}}=0,17$ & 0,0116 & 0,8206 \\
\hline C18:1 c9 & 14,9196 & 11,7878 & 13,4570 & 13,3620 & 12,1588 & $\hat{\mathrm{Y}}=13,13$ & 0,0374 & 0,7491 \\
\hline $\mathrm{C} 18: 1$ trans & 1,2354 & 1,0648 & 1,1278 & 1,0404 & 1,0028 & $\hat{\mathrm{Y}}=1,09$ & 0,5526 & 0,7261 \\
\hline C18:1 c11 & 0,5998 & 0,4972 & 0,5196 & 0,4516 & 0,5388 & $\hat{Y}=0,52$ & 0,0407 & 0,9791 \\
\hline C18:1 c12 & 0,3292 & 0,2676 & 0,2862 & 0,2486 & 0,2816 & $\hat{\mathrm{Y}}=0,28$ & 0,0244 & 0,9281 \\
\hline C18:1 c13 & 0,1824 & 0,1512 & 0,1602 & 0,1222 & 0,1236 & $\hat{\mathrm{Y}}=0,14$ & 0,0134 & 0,9616 \\
\hline C18:1 t16 & 0,1130 & 0,0998 & 0,1010 & 0,1074 & 0,0800 & $\hat{\mathrm{Y}}=0,10$ & 0,0114 & 0,3753 \\
\hline C18:1 c15 & 0,0342 & 0,0280 & 0,0324 & 0,0326 & 0,0236 & $\hat{Y}=0,03$ & 0,0056 & 0,5101 \\
\hline C20:1 & 0,0066 & 0,0074 & 0,0104 & 0,0082 & 0,0086 & $\hat{\mathrm{Y}}=0,00$ & 0,0019 & 0,7322 \\
\hline C22:1n9 & 0,0050 & 0,0074 & 0,0066 & 0,0058 & 0,0088 & $\hat{\mathrm{Y}}=0,00$ & 0,0006 & 0,8151 \\
\hline C24:1 & 0,3658 & 0,3790 & 0,4600 & 0,4568 & 0,3658 & $\hat{\mathrm{Y}}=0,40$ & 0,0007 & 0,9638 \\
\hline$\Sigma$ AGPI3 & 1,9940 & 1,8666 & 2,1818 & 2,1858 & 2,0360 & $\hat{\mathrm{Y}}=2,05$ & 0,0032 & 0,6237 \\
\hline $\mathrm{C} 18: 2 \mathrm{c} 9 \mathrm{c} 12$ & 1,2146 & 1,1740 & 1,3446 & 1,4122 & 1,3210 & $\hat{\mathrm{Y}}=1,29$ & 0,0436 & 0,5631 \\
\hline C18:3 n6 & 0,0038 & 0,0074 & 0,0100 & 0,0104 & 0,0114* & $\hat{\mathrm{Y}}=0,008$ & 0,0014 & 0,5188 \\
\hline $\mathrm{C} 18: 3 \mathrm{n} 3$ & 0,2610 & 0,2234 & 0,2866 & 0,2740 & 0,2656 & $\hat{\mathrm{Y}}=0,26$ & 0,0106 & 0,5984 \\
\hline C18:2 c9t11 & 0,3284 & 0,3028 & 0,3374 & 0,3160 & 0,2586 & $\hat{\mathrm{Y}}=0,30$ & 0,0138 & 0,6573 \\
\hline C20:2 & 0,0044 & 0,0066 & 0,0012 & 0,0034 & 0,0052 & $\hat{Y}=0,004$ & 0,0009 & 0,7490 \\
\hline $\mathrm{C} 20: 3 \mathrm{n} 6$ & 0,0104 & 0,0120 & 0,0206 & 0,0140 & 0,0156 & $\hat{Y}=0,01$ & 0,0018 & 0,5695 \\
\hline C20:4 n6 & 0,0826 & 0,0682 & 0,1050 & 0,0878 & 0,0882 & $\hat{\mathrm{Y}}=0,08$ & 0,0059 & 0,1297 \\
\hline $\mathrm{C} 22: 2$ & 0,0012 & 0,0026 & 0,0012 & 0,0006 & 0,0006 & $\hat{\mathrm{Y}}=0,001$ & 0,0004 & 0,5525 \\
\hline $\mathrm{C} 20: 5 \mathrm{n} 3$ & 0,0232 & 0,0192 & 0,0222 & 0,0158 & 0,0146 & $\hat{\mathrm{Y}}=0,01$ & 0,0017 & 0,5299 \\
\hline $\mathrm{C} 22: 5$ & 0,0466 & 0,0390 & 0,0526 & 0,0484 & 0,0510 & $\hat{\mathrm{Y}}=0,04$ & 0,0024 & 0,7744 \\
\hline $\mathrm{C} 22: 6 \mathrm{n} 3$ & 0,0178 & 0,0114 & 0,0004 & 0,0032 & 0,0042 & $\hat{\mathrm{Y}}=0,007$ & 0,0436 & 0,2348 \\
\hline
\end{tabular}

*Diferem da testemunha pelo teste Dunnett; 1. ácidos graxos saturados; 2. ácidos graxos monoinsaturados; 3. ácidos graxos poli-insaturados. 
Tabela 4. Índice de aterogenicidade (IA), índice de trombogenecidade (IT), relação hiper/hipocolesterolêmico $(\mathrm{h} / \mathrm{H})$, ácidos graxos desejáveis (AGD, relação de ácidos graxos poliinsaturados/ácidos graxos saturados (AGP/AGS), somatório de ácidos graxos $\omega 6$ e relação $\omega 6 / \omega 3$ do queijo minas frescal e do leite de vacas F1 Holandês x Zebu alimentadas com níveis crescentes de casca de banana na dieta e suas respectivas equações de regressão (ER), erro-padrão da média (EPM) e valor real de $\mathrm{P}(\mathrm{Pr}>\mathrm{Fc})$

\begin{tabular}{|c|c|c|c|c|c|c|c|c|}
\hline \multirow{3}{*}{ Variáveis } & \multicolumn{5}{|c|}{ Dietas experimentais } & \multirow[b]{2}{*}{ ER } & \multirow[b]{2}{*}{ EPM } & \multirow[b]{2}{*}{$\mathrm{Pr}>\mathrm{Fc}$} \\
\hline & 0 & 15 & 30 & 45 & 60 & & & \\
\hline & \multicolumn{8}{|c|}{ Leite } \\
\hline IA & 4,00 & 4,39 & 4,33 & 4,09 & 4,00 & $\hat{\mathrm{Y}}=4,16$ & 0,0828 & 0,8773 \\
\hline IT & 2,46 & 2,59 & 2,75 & 2,67 & 2,50 & $\hat{\mathrm{Y}}=2,59$ & 0,0542 & 0,7209 \\
\hline $\mathrm{H} / \mathrm{H}$ & 0,48 & 0,50 & 0,44 & 0,46 & 0,51 & $\hat{\mathrm{Y}}=0,48$ & 0,0545 & 0,6241 \\
\hline AGD & 31,93 & 32,22 & 30,40 & 31,00 & 32,80 & $\hat{\mathrm{Y}}=31,67$ & 0,4309 & 0,7391 \\
\hline AGP/AGS & 0,027 & 0,024 & 0,024 & 0,026 & 0,030 & 1 & 0,0012 & 0,0334 \\
\hline$\omega 6$ & 1,41 & 1,26 & 1,32 & 1,44 & 1,72 & 2 & 0,0847 & 0,0054 \\
\hline \multirow[t]{2}{*}{$\omega 6 / \omega 3$} & 3,88 & 4,88 & 4,22 & 4,84 & $5,85^{*}$ & 3 & 0,0178 & 0,0123 \\
\hline & \multicolumn{8}{|c|}{ Queijo } \\
\hline IA & 4,27 & 5,16 & 4,38 & 4,54 & 5,35 & $\hat{\mathrm{Y}}=4,74$ & 0,2165 & 0,6400 \\
\hline IT & 2,49 & 3,19 & 2,60 & 2,64 & 3,45 & $\hat{\mathrm{Y}}=2,87$ & 0,1885 & 0,5007 \\
\hline $\mathrm{H} / \mathrm{H}$ & 0,51 & 0,39 & 0,46 & 0,45 & 0,41 & $\hat{\mathrm{Y}}=0,44$ & 0,1638 & 0,7746 \\
\hline AGD & 32,27 & 28,66 & 30,14 & 29,65 & 28,98 & $\hat{\mathrm{Y}}=29,94$ & 0,6365 & 0,9475 \\
\hline AGP/AGS & 0,027 & 0,024 & 0,028 & 0,028 & 0,026 & $\hat{\mathrm{Y}}=0,02$ & 0,0009 & 0,6374 \\
\hline$\omega 6$ & 1,36 & 1,30 & 1,53 & 1,57 & 1,49 & $\hat{\mathrm{Y}}=1,45$ & 0,0083 & 0,5276 \\
\hline$\omega 6 / \omega 3$ & 4,5455 & 7,2694 & 5,0141 & 5,4107 & 5,2909 & $\hat{\mathrm{Y}}=4,42$ & 0,0316 & 0,6949 \\
\hline
\end{tabular}

Os ácidos graxos considerados prótrombogênicos são o láurico, o mirístico e o palmítico, enquanto os ácidos graxos poliinsaturados são admitidos com diferentes potenciais para compor o índice, sendo os monoinsaturados e ácidos graxos ômega 6 considerados com menor potencial comparados aos ácidos graxos ômega 3 (Costa et al., 2008). Esse índice não foi influenciado pelas dietas, sendo o valor médio observado para o leite de 2,59 e 2,87 para o queijo. Mourthé et al. (2015) encontraram reduções lineares quando inseriram grão de soja tostado em diferentes níveis na dieta de vacas leiteiras Holandês x Gir, ao observarem índices de trombogenecidade no leite, que variaram de 4,12 a 2,4 .

A relação de ácidos graxos hipo e hipercolesterolêmicos não apresentou efeito das dietas. Em contraste, Mourthé et al. (2015) encontraram incrementos lineares, com valores que variaram de 0,49 a 0,97 no leite quando grão de soja tostado foi adicionado à dieta de vacas. Não foi observado efeito das dietas sobre os ácidos graxos desejáveis (AGD) do leite e do queijo. $\mathrm{O}$ ácido esteárico é um dos ácidos graxos utilizados para o cálculo desse índice e, apesar de ser saturado, é considerado um ácido graxo neutro sob o ponto de vista do perfil lipídico, já que pode ser convertido em ácido oleico, o qual, por sua vez, contribui para a redução nos níveis de LDL-colesterol e, por conseguinte, diminui os riscos de aparecimento de doenças cardiovasculares (Costa et al., 2008). Já a razão entre ácidos graxos poli-insaturados e saturados (AGP/AGS) no leite apresentou efeito das dietas, sendo o valor mínimo observado quando $23,3 \%$ de casca de banana foram introduzidos na dieta em substituição à silagem de sorgo, ponto a partir do qual houve incremento nessa relação até o nível máximo testado. Apesar do efeito significativo, os valores encontrados estão abaixo do recomendado pelo Departamento de Saúde e Seguridade Social dos EUA, que recomenda valores acima de 0,45 na dieta. Cabe ressaltar que essa relação considera que todos os ácidos graxos saturados promovem aumento do LDLcolesterol, além de ocultarem os AGMI. Nesse sentido, a avaliação do aspecto funcional dos ácidos graxos sobre o metabolismo seria mais importante (Caldeira et al., 2010). Ressalta-se, ainda, que a recomendação do órgão de saúde dos EUA refere-se à dieta total e não dos alimentos individualmente. Não houve efeito das dietas sobre esse índice nos queijos obtidos, sendo a média geral encontrada de 0,02 . 
Os ácidos graxos $\omega 6$, bem como a relação $\omega 6 / \omega 3$ no leite, sofreram efeitos linear e quadrático das dietas, respectivamente. A substituição de $23,56 \%$ de casca de banana na dieta proporcionou menores relações entre esses ácidos graxos no leite. Além disso, a relação $\omega 6 / \omega 3$ na dieta com $60 \%$ de substituição de silagem de sorgo por casca de banana foi significativamente maior quando comparada com a relação obtida no leite de vacas alimentadas com a dieta testemunha. $\mathrm{O}$ principal ácido graxo da série n-6 é o linoleico (C18:2n6), a partir do qual os demais são sintetizados por uma série de enzimas que promovem o alongamento, a dessaturação e a $\beta$-oxidação das cadeias carbônicas (Martin et al., 2006). Os dados observados neste estudo para os teores do ácido linoleico no leite sustentam o fato de que os teores desse ácido graxo foram responsáveis pelo aumento linear obtido para o ácido graxo ômega 6 , já que os demais ácidos graxos pertencentes à série apresentam uma limitação na eficiência de sua transferência para a gordura do leite. Há uma negativa correlação entre os ácidos graxos da série ômega 6 com risco de doenças cardiovasculares (Ferrucci et al., 2006). Segundo Martin et al. (2006), o intervalo de convergência para a relação $\omega 6 / \omega 3$ é de $4: 1$ a $5: 1$, indicando que os valores obtidos neste estudo estão dentro da faixa considerada ideal pelos órgãos de saúde de diferentes países.

\section{CONCLUSÕES}

A substituição de até $60 \%$ da silagem de sorgo por casca de banana seca ao sol na dieta de vacas F1 Holandês x Zebu diminui os teores de ácidos graxos de cadeia ímpar e ramificada (C15:0iso e C16:0iso) e aumenta os teores dos ácidos graxos poli-insaturados totais, ácido linoleico, CLA (C18:2cis9trans11) e ácido araquidônico no leite. A relação $\omega 6 / \omega 3$ no leite é maior nas dietas contendo $60 \%$ de casca, melhorando as características nutricionais do produto. $\mathrm{O}$ perfil de ácidos graxos e as características nutricionais do queijo Minas frescal não são influenciados pela inclusão de casca de banana seca ao sol na dieta das vacas.

\section{AGRADECIMENTOS}

À Fapemig, pelo auxílio financeiro ao projeto; ao CNPq e à Capes, pelo auxílio com bolsas; ao INCT-Ciência Animal.

\section{REFERÊNCIAS}

BASSETT, M.C.C.; EDEL, A.L.; PATENAUDE, A.F. et al. Dietary vaccenic acid has antiatherogenic effects in LDL LDLr-/- mice. J. Nut. Dis., v.140, p.18-24, 2010.

BENCHAAR, C.; MCALLISTER, T.A.; CHOUINARD, P.Y. Digestion, ruminal fermentation, ciliate protozoal populations, and milk production from dairy cows fed cinnamaldehyde, quebracho condensed tannin, or Yucca schidigera saponin extracts. J. Dairy Sci., v.91, p.4765-4777, 2008.

CALDEIRA, L.A.; FERRÃO, S.P.B.; FERNANDES, S.A.A. et al. Índices de qualidade nutricional da fração lipídica do leite de búfalas da raça Murrah produzido em diferentes fases de lactação. Rev. Inst. Adolfo Lutz, v.69, p.545-554, 2010.

CHILLIARD, Y.; GLASSER, F.; FERLAY, A. et al. Diet, rumen biohydrogenation and nutritional quality of cow and goat milk fat. Eur. J. Lipid Sci. Technol., v.109, p.828-855, 2007.

COSTA, R.G.; FERNANDES, M.F.; QUEIROGA, R.C.R.E. Características químicas e sensoriais do leite de cabras Moxotó alimentadas com silagem de maniçoba. Rev. Bras. Zootec., v.37, p.694-702, 2008.

DILZER, A.; PARK, Y. Implication of conjugated linoleic acid (CLA) in human health. Crit. Rev. Food Sci. Nutr., v.52, p.488-513, 2012.

EMAGA, T.H.; ANDRIANAIVO, R.H.; WATHELET, B. et al. Effects of the stage of maturation and varieties on the chemical composition of banana and plaintais peels. Food Chem., v.103, p.590-600, 2007.

FERRUCCI, L.; CHERUBINI, A.; BANDINELLI, S. et al. Relationship of plasma polyunsaturated fatty acids to circulating inflammatory markers. J. Clin. Endoc. Metab., v.91, p.439-446, 2006.

FURTADO, M.M.; LOURENÇO NETO, J.P.M. (Eds.). Tecnologia de queijos: manual técnico para produção industrial de queijos. São Paulo: Dipemar, 1994. 118p. 
GARCÍA， G.A.G.; REIS, R.B.; PEREIRA, A.B.D. et al. Produção e composição do leite de vacas em pastagem de capim-elefante (Pennisetum purpureum) suplementado com diferentes fontes de carboidratos. Arq. Bras. Med. Vet. Zootec., v.62, p.875-882, 2010.

LAHLOU, M.N.; KANNEGANTI, R.; MASSINGILL, L.J. et al. Grazing increases the concentration of CLA in dairy cow milk. Animal, v.8, p.1191-1200, 2014.

LANIER, J.S.; CORL, B.A. Challenges in enriching milk fat with polyunsaturated fatty acids. J. Anim. Sci. Biotechnol., v.6, p.26, 2015.

LUCATTO, J.N.; MENDONÇA, S.N.T.G.; DRUNKLER, D.A. Ácido linoleico conjugado: estrutura química, efeitos sobre a saúde humana e análise em lácteos. Rev. Inst. Laticinio Cândido Tostes, v.69, p.199-211, 2014.

MAHOPATRA, D.; MISHRA, S.; SUTAR, N. Banana and its by-product utilization: an overview. J. Sci. Ind. Res., v.69. p.323-329, 2010.

MARTIN, C.A.; ALMEIDA, V.V.; RUIZ, M.R. et al. Ácidos graxos polinsaturados ômega-3 e ômega-6: importância e ocorrência em alimentos. Rev. Nutr., v.19, p.761-770, 2006.

MENEZES, L.F.G.; KOZLOSKI, G.V.; RESTLE, J. et al. Profile of ingested fatty acids and in the duodenal digest of steers fed different diets. Rev. Bras. Zootec., v.39, p.2502-2511, 2010 .

MOURTHÉ, M.H.F.; REIS, R.B.; GAMA, M.A.S. et al. Perfil de ácidos graxos do leite de vacas Holandês x Gir em pastagem de capimmarandu suplementado com quantidades crescentes de grão de soja tostado. Arq. Bras. Med. Vet. Zootec., v.67, p.1150-1158, 2015.

NUDDA, A.; BATTACONE, G.; BOAVENTURA NETO, O. et al. Feeding strategies to desing the fatty acid profile of sheep Milk and cheese. Rev. Bras. Zootec., v.43, p.445$456,2014$.
OLIVEIRA, M.L.M.; NUNES-PINHEIRO, D.C.S. Biomarcadores celulares e moleculares envolvidos na resposta imune-inflamatória modulada por ácidos graxos insaturados. Acta Vet. Bras., v.7, p.113-124, 2013.

REIS, A.M.; COSTA, M.R.; COSTA, R.G. et al. Efeito do grupo racial e do número de lactações sobre a produtividade e a composição do leite bovino. Semin. Cienc. Agrar., v.33, Supl.2, p.3421-3436, 2012.

RIBEIRO, C.G.S.; LOPES, F.C.F.; GAMA, M.A.S. et al. Desempenho produtivo e perfil de ácidos graxos do leite de vacas que receberam níveis crescentes de óleo de girassol em dietas à base de capim-elefante. Arq. Bras. Med. Vet. Zootec., v.66, p.1513-1521, 2014.

SANTOS, R.D.; GAGLIARDI, A.C.M.; XAVIER H.T. et al. Sociedade Brasileira de Cardiologia. I Diretriz sobre o consumo de gorduras e saúde cardiovascular. Arq. Bras. Cardiol. v.100, Supl.3, p.1-40, 2013.

SCHWINGSHACKL, L.; HOFFMANN, G. Dietary fatty acids in the secondary prevention of coronary heart disease: a systematic review, meta-analysis and meta-regression. BMJ Open, v.4, p.1-9, 2014.

SIRI-TARINO, P.W.; SUN, Q.; HU, F.B.; KRAUSS, R.M. Saturated fatty acids and risk of coronary heart disease: modulation by replacement nutrients. Curr. Atheroscler. Rep., v.12, p.384-390, 2010.

TORAL, P.G.; HERVÁS, G.; BICHI, E. et al. Tannins as feed additives to modulate ruminal biohydrogenation: effects on animal performance, milk fatty acid composition and ruminal fermentation in dairy ewes fed a diet containing sunflower oil. Anim. Feed Sci. Technol., v.164, p.199-206, 2011.

ULBRICHT, T.L.V.; SOUTHGATE, D.A.T. Coronary heart disease: seven dietary factors. Lancet, v.338, p.985-992, 1991.

VLAEMINCK, B.; FIEVEZ, V.; TAMMINGA, S. et al. Milk odd- and branched-chain fatty acids in relation to the rumen fermentation pattern. $J$. Dairy Sci., v.89, p.3954-3964, 2006. 